\section{Zauberwort Konvergenz}

\author{
von Christopher Coenen, ITAS
}

Viel heiße Luft, konzeptionelle Unklarheit, eine bedenkliche ideologische Schlagseite und politische Irrelevanz: So lässt sich pointiert zusammenfassen, wie eine geballte Kritik seit Mitte dieses Jahrzehnts den Diskurs zu "Converging Technologies“" (CT) - und speziell die Ideen der ersten, zur Jahrtausendwende in den USA gestarteten CTInitiative - charakterisiert hat. Dennoch erweist sich das neue Konvergenzkonzept als erstaunlich vital. In einer weitgehend von seiner technikvisionären Aufladung gereinigten Version hat es sich in einigen Bereichen der EU-Forschungspolitik etabliert, in verschiedenen sozial- und geisteswissenschaftlichen Disziplinen erfolgte eine Öffnung hin zum „CT-Diskurs“, und auch in Aktivitäten zur öffentlichen Vermittlung oder Diskussion von Naturwissenschaft und Technik gewinnt der Begriff der Konvergenz an Bedeutung. Die Herausforderungen, die sich hier für die sozial- und geisteswissenschaftliche Begleitforschung und die Technikfolgenabschätzung (TA) ergeben haben, sind vielfältig. Vorrangig erscheint derzeit jedoch, ein klareres Bild von diesem sich ausweitenden Diskurs zu erlangen und eine Position kritischer Distanz zurückzugewinnen.

Seit Mitte dieses Jahrzehnts konnte man zunehmend den Eindruck gewinnen, dass das Thema "CT" tot und weder für eine vertiefte akademische Auseinandersetzung geeignet noch im forschungs- und technologiepolitischen Kontext relevant sei: Die Kritik an „spekulativer Ethik“ traf den durch „Human-Enhancement"-Visionen geprägten CT-Diskurs mit Wucht, verschiedene TA- und Foresight-Studien kamen - u. a. hinsichtlich der prominentesten Konvergenzkonzepte und einiger der meist diskutierten CTForschungsbereiche und -Anwendungen - zu ernüchternden Ergebnissen ${ }^{1}$, und die in den Publikationen der ersten Inititiative zu CT - der

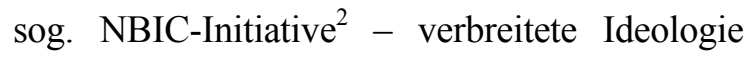
wurde aus den verschiedensten Perspektiven kritisiert, wobei die Visionen einer technischen „Verbesserung“ des Menschen im Fokus standen (z. B. Grunwald 2007). Zwar war so ,in sehr kurzer Zeit eine eigene Diskursgemeinschaft mit kanonischen Referenzen" (Kogge 2008) entstanden, letztendlich schien sich der ganze Konvergenzdiskurs aber als „hype“ zu entpuppen, als ein (nur mäßig erfolgreicher) Versuch, extrem hohe Erwartungen an bestimmte wissenschaftlich-technische Entwicklungen zu schüren.

Im Folgenden soll eine profilierte aktuelle Ausstellung zum Anlass genommen werden, diese Einschätzungen mit einem Fragezeichen $\mathrm{zu}$ versehen und einige Bemerkungen zur Bedeutung des Konvergenzdiskurses - und zu den Herausforderungen für die CT-Begleitforschung in diesem - zu machen. ${ }^{3}$

\section{Aufbruch in eine konvergenz- technologisch geprägte Zukunft?}

Karlsruhe, im Sommer 2009: Auf dem hintersten Gleis des Bahnhofs Durlach steht der auf Deutschlandtour befindliche Ausstellungszug „Expedition Zukunft“. Gefördert vom Bundesministerium für Bildung und Forschung und realisiert durch die Max-Planck-Gesellschaft (MPG) soll er zeigen, wie Forschung und Technik in den kommenden Jahren unser Leben verändern werden. Das Projektteam der MPG konnte dafür in seiner Arbeit auf die Unterstützung großer deutscher Forschungsorganisationen und Firmen sowie vieler Hochschulen bauen. Anstelle eines Katalogs ist am Ausgang der Ausstellung eine maßgeblich vom Projektteam mitgestaltete Sonderausgabe der Zeitschrift „Spektrum der Wissenschaft" käuflich erhältlich, in der zu jedem Themenblock jeweils ein kurzer Einführungstext sowie ein einschlägiger Artikel in Zweitveröffentlichung enthalten ist.

Trotz des schwülen Sommerwetters stehen die Interessierten geduldig eine Viertelstunde Schlange, um den Zug danach von vorne bis hinten durchqueren zu können. $\mathrm{Zu}$ mindest an diesem Tag erreicht das Projekt seine vorrangige Zielgruppe, nämlich Jugendliche und Kinder. Diese sollen laut Bundesministerin Annette Schavan durch den Ausstellungszug für Forschung „fasziniert" werden, weil „die Wissenschaft" nachfolgenden Generationen einen „spannenden Auftrag“ biete, „nämlich der Welt von übermorgen den Weg zu bereiten" (SdW 2009, S. 3).

In jedem der zwölf Wagen wird ein Themenblock behandelt. Im ersten geht es um die Frage „Was kommt auf uns zu?“. Als relevante Fragen werden beispielsweise der Klimawandel 
und die Welternährung genannt, aber z. B. auch, ob schon im Jahr 2050 alle Gehirne auf eine Festplatte passen, ob wir die Liebe zu einer Person durch Medikamente sicherstellen und $a b$ wann wir uns in unsere Roboter verlieben werden. Der zweite Wagen ist dem ,woher + wohin" gewidmet, der Entstehung und Entwicklung des Universums und des Lebens. Der zugehörige Artikel in der Sonderausgabe berichtet von der ,hindernisreiche[n] Suche nach der Weltformel", mit der das gesamte physikalische Universum eines Tages mittels einer einzigen fundamentalen Theorie beschreibbar sein soll.

Der dritte Wagen steht unter dem Motto „bio + nano“ und dient dazu, die Konvergenz der Nano- und Biowissenschaften zu veranschaulichen. Die Texte der Organisatoren zum dritten Wagen kombinieren die vorherrschenden „Narrative“ zur Synthetischen Biologie und zur Nanotechnologie: In gewisser Hinsicht sei die „Vision“ Richard Feynmans vom Manipulieren und sozusagen "Sehen“" einzelner Atome bereits realisiert, sein „Traum wahr geworden“: Noch seien wir zwar nicht ,in der kleinsten aller Welten angekommen“, es gebe noch „viel Spielraum nach unten", es stelle sich aber schon jetzt die Frage, wann Forscher ,einzelne Atome und Moleküle perfekt und nach Bedarf zusammensetzen" können werden. Eines der Exponate soll das zukünftige Arbeiten auf dieser Ebene spielerisch verdeutlichen: In kindgerechter Höhe ist ein durchsichtiger Kasten mit zwei Öffnungen aufgebaut, in dem zusammengeklumpt magnetische Kugeln in roter oder grüner Farbe liegen. In die Öffnungen sind kleine Stoffsäcke eingehängt, in die zwei Besucher je eine Hand stecken, um dann gemeinsam die Kugeln neu kombinieren zu können - da die Stoffsäcke nicht magnetisch sind, bleibt dabei das von Richard Smalley gegen den Nanofuturisten Eric Drexler vorgebrachte „Klebrige-Finger“-Argument unberücksichtigt, dass nämlich durch zwischenmolekulare oder -atomare Kräfte Kleinstteilchen an dem Instrument „hängenbleiben“ würden. Ein großer Teil der weiteren Exponate im dritten Wagen ist der Synthetischen Biologie gewidmet. Dementsprechend heißt es im Begleittext des MPG-Projektteams, dass die „Konvergenz von Nano- und Biowissenschaften [...] unübersehbar" voranschreite und die Synthetische Biologie die ,neue Nanotechnologie“" sei. Der zugehörige Beitrag in der Sonderausgabe entspricht dem Stand des Diskurses und geht, u. a. durch ein Interview mit Markus Schmidt, auch auf Themen der TA, Technikethik und Sicherheitsbewertung in diesem Forschungsfeld ein - Aspekte, die in dem Wagen selbst keine Rolle spielen.

Der vierte Wagen hat das Motto ,info + cogno" und stellt im Untertitel die Frage, ob „das Gehirn“ als ,ein intelligenter Computer“ zu begreifen sei. Die Engführung von Hirnforschung und Computertechnologie wird bei diesem Themenblock unter Berücksichtigung all jener zentralen Aspekte des Themas behandelt, die auch den CT-Spezialdiskurs sowie die Debatte über "human enhancement" prägen. Die Vision der Erschaffung eines künstlichen Gehirns findet ebenso Erwähnung wie die Vorstellung, dass Robotern „Leben eingehaucht“ werden könnte, wobei Leben als die Fähigkeit verstanden wird, „wie wir Menschen Information [zu] verarbeiten und autonom [zu] handeln". Informationen zu Hirn-Computer-Schnittstellen nehmen breiten Raum ein wie auch die Erwartung, dass bald durch „fortschrittlichste Computertechnik“" (z. B. Bio- und Quantencomputer) der ,Sprung zur Künstlichen Intelligenz“ gelingen werde. Es wird gefragt, ob sich der freie Wille, das Mitgefühl, die moralische Verantwortung, die Entscheidungsfindung und das Verliebtsein vollständig durch biochemische Vorgänge in Nervenzellen erklären lassen werden. Zudem heißt es, dass in Zukunft Neurotechnologien zur Verfügung stehen werden, deren Anwendungen „,vom Gedankenlesen über Lügendetektion bis hin zur Marktforschung" reichen. Die Organisatoren betonen zudem, dass bei einer Entschlüsselung des neuralen Codes der Mensch sich selbst und sein Handeln begreifen, aber auch „seine geistigen Leistungen oder Charakterzüge" beeinflussen können werde. Im zugehörigen Artikel der Sonderausgabe wird dann vor allem auf die einschlägigen Themen der Debatte über „cognitive enhancement" eingegangen, vom „Hirndoping“ mit Medikamenten bis hin zu den ambitionierten neurotechnologischen Projekten der US-Militärforschung (vgl. zu dieser Debatte: STOA 2009).

\section{Von Visionen zu Anwendungen?}

Kombiniert man das englische Motto des dritten $($, nano + bio“) mit dem des vierten Wagens 
(,info + cogno") und kürzt das Ergebnis ab, ergibt dies das zentrale Akronym der Konvergenzdebatte: „NBIC“. Im Unterschied zur USamerikanischen NBIC-Initiative führt „Expedition Zukunft" das relativ neue Thema Synthetische Biologie ein, und die Ausstellung setzt auch beim ,human enhancement", bei den Neurotechnologien und der Computertechnik geringfügig andere Akzente, $\mathrm{z}$. B. durch die relativ starke Berücksichtigung der Themen ,Lügendetektion“ und „Quantencomputer" und die weitgehende Nichterwähnung von militärischen Einsatzmöglichkeiten konvergierender Technologien. Das thematische Spektrum des Ausstellungszugs ist aber weitgehend deckungsgleich mit dem der Publikationen der NBIC-Initiative, und es lässt sich bei Ersterem eine ähnlich starke Faszination durch die Visionen einer ,starken“ Künstlichen Intelligenz und der Schaffung eines künstlichen Gehirns feststellen. Ebenfalls ähnlich wie bei der NBIC-Initiative (und zum Teil bei der US-amerikanischen nationalen Nanotechnologieinitiative) ist der Drexlerianische Nanofuturismus zwar tabuiert, die im Rückgriff auf Feynmans Rede von 1959 entwickelten Visionen zur Nanotechnologie sind aber sehr weitreichend.

Die Wagen zum „woher + wohin“ und zur NB- sowie IC-Konvergenz sollen die Besucher auf Entwicklungen in der Grundlagenforschung einstimmen, durch die allen anderen Bereiche (vor allem Medizin, Ernährung, Kommunikationstechnik, Arbeitswelt und Privatleben) ,zusätzliche Impulse" verliehen würden (SdW 2009, S. 7). Um solche breiten Anwendungsbereiche geht es dann in den Wagen 5 bis 10, die den „Weg in die digitale Gesellschaft" skizzieren (5), ,[i]nnovative Materialien und die Fabrik der Zukunft" darstellen (6), nach der Möglichkeit einer „Welt ohne Krankheiten“ fragen (7) und die Themen Welternährung (8), nachhaltige Energieversorgung (9) sowie Mobilität (10) behandeln.

Im fünften Wagen wird die Entstehung einer ,digitale[n] Welt" konstatiert, in der sich „Kreativität und Innovation auf neue Weise entfalten“ könnten. Schon jetzt steigere die „digitale Vernetzung der Vielen“ die „Kreativität jedes Einzelnen“, wir lebten in ,beschleunigten Zeiten“ und seien „Zeugen einer Explosion der menschlichen Kreativität". Auf der Grundlage „massive[r] Investitionen in For- schung und Entwicklung“, in ,intensive[r] Kooperation“ und im „harte[n] Wettbewerb“ werde die Konvergenz von Nano-, Bio-, Informations- und Neurotechnologien völlig neue Methoden und Anwendungen ermöglichen. Unter den Exponaten (u. a. zum semantischen Netz, zur ,systematischen Vermessung der Welt" von der kosmischen zur Nanoebene und zur globalen Forschungslandschaft) stößt bei den jungen Besuchern vor allem die Leihgabe der Stiftung „One Laptop per Child“ auf starkes Interesse, ein Exemplar des sog. „HundertDollar-Laptops“, dessen Funktionen zum Teil getestet werden können. Damit sich die Wissenskluft zwischen reichen und armen Ländern verringere, benötigten überdies ,gerade die am wenigsten entwickelten Länder Zugang zum Internet". 5 Bezüglich der Zukunft des Netzes wird u. a. die Frage aufgeworfen, ob ein ,globales Gehirn" im Entstehen begriffen sei - ein alter technikfuturistischer Topos, den auch die NBIC-Initiative aufgegriffen hatte -, und es wird prognostiziert, dass uns vernetzte Systeme „auf Schritt und Tritt begleiten“ sowie „Dinge und Dienste um uns herum [...] miteinander und mit uns sprechen" werden. Der Wagen 5 leistet so, durchaus ähnlich wie es aus dem CT-Diskurs schon bekannt war, die rhetorische Verknüpfung der internetzentrierten Narrative über Globalisierung, Vernetzung, Beschleunigung und Virtualisierung mit der Verheißung einer radikal innovativ wirkenden NBIC-Konvergenz.

In den Wagen 6 bis 10 spielt das Konvergenzkonzept hingegen keine Rolle mehr. Sehr weit reichende Visionen finden sich aber z. B. im Wagen 7, der den Titel „Wird es eine Welt ohne Krankheiten geben?" trägt. Und an das Ethikverständnis der NBIC-Initiative erinnert die Feststellung im achten Wagen, dass ,genetisch optimiertes Getreide“ zwar längst angebaut werde, die Anwendung der entsprechenden Technologien aber ,schneller“" verlaufe als ,ihre ethisch-moralische Integration".

Es sind vor allem die Wagen 5 bis 10 der Ausstellung, durch die deren Anspruch eingelöst wird, Blicke in eine Welt von übermorgen $\mathrm{zu}$ ermöglichen - in eine Welt, in der Wissenschaft und Technik in verschiedenen Alltags- und Wirtschaftsbereichen sowie zur Lösung gravierender globaler Probleme eingesetzt werden. Hier kann sich der Reiz einer Vorausschau entfalten, die von der heutigen Situation ausgeht 
und auf unrealistische oder ethisch problematische Visionen weitgehend verzichtet.

\section{Zur Berücksichtigung ethischer Aspekte}

Der elfte Wagen, an dessen Ende sich der Ausgang der Ausstellung befindet, steht unter dem Motto „natürlich. künstlich“, der Untertitel lautet „Die Zukunft des Menschen“. In ihm soll es nun nicht nur um vielversprechende Möglichkeiten gehen, die sich durch Wissenschaft und Technik bieten, sondern auch um ,ethische Grenzen“" der Entwicklung. Die Konvergenzbegrifflichkeit taucht nun wieder auf: „Genetik, Kognitionsforschung, Nano- und Informationstechnologien", heißt es im Einführungstext, ermöglichten „Eingriffe in die künftige Entwicklung des Menschen“. Deren Konsequenzen sollen in Wagen 11 ,namhafte Wissenschaftler, Gelehrte und Philosophen" aufzeigen.

Die Informationen zu deren Ansichten werden in einem Ambiente bereitgestellt, das eine Besucherin zu dem Ausruf „Wie in der Disco!" motiviert: Wechselnd stark beleuchtete quadratische Platten von unterschiedlicher Farbe, in einem Gittermuster angeordnet, dominieren die Wände des Wagens. Dazu ertönt, wie auch anderswo im Ausstellungszug, leise wabernde „Techno“-Musik. Zwischen die farbigen Quadrate sind in deutlich geringerer Zahl weitere Quadrate in kleinen Gruppen eingefügt, die den verschiedenen NBIC-Feldern gewidmet sind. Ausschnitte aus Texten oder Interviewaussagen der Experten stehen dort, zumeist in schwarz-weiß gehalten, jeweils neben einem Quadrat, auf dem man den jeweiligen Experten, ebenfalls in schwarz-weiß, sprechen sieht. Um diese informativen Kerne herum gelagert sind einige Quadrate mit ganz überwiegend bunten und zum Gesamtambiente des Wagens passende Visualisierungen und Bildern zu wissenschaftlichen Erkenntnissen oder technischen Entwicklungen. Die Informationen zu den Stellungnahmen der Experten fallen somit ins Auge, stehen dabei aber in einem eigentümlichen Kontrast sowohl zu den Illustrationen ihrer jeweiligen Themengebiete als auch insgesamt zur Vielzahl der einfarbigen, aber bunt gemischten Platten, die - augenscheinlich abgestimmt zum Rhythmus der Musik - mal heller, mal dunkler leuchten. Gerd Karl Binnig äußert sich hier zu der Frage, ob die Nanotechnologie eine neue Form der Evolution begründen wird, Julian NidaRümelin macht sich Gedanken über die Rolle einer humanistischen Ethik angesichts der Herausforderungen durch die Hirnforschung, Thomas Christaller weist auf die Ideen(vor)geschichte der Vision intelligenter Roboter hin, und Alfred Nordmann erläutert einen seiner Beiträge zur Entmystifizierung der Nanotechnologie. Von Niels Birbaumer findet sich, um ein weiteres Beispiel zu nennen, die Prognose, dass in naher Zukunft die Menschen ,eine Art Symbiose" mit an menschliche Gehirne angeschlossenen Computern eingehen werden.

Wer unter den Besuchern sich danach vertiefend zu solchen Themen oder zu den ,ethischen Grenzen" der wissenschaftlich-technischen Entwicklung informieren will, wird womöglich zur erwähnten Sonderausgabe von „Spektrum der Wissenschaft" greifen. Dort findet sich zu Wagen 11 allerdings bloß ein Artikel des Erfinders und einflussreichen Technikfuturisten Ray Kurzweil. Sein Beitrag (Kurzweil 2009) behandelt, mit dem für ihn üblichen Enthusiasmus, die Idee einer „Singularität“, also die quasireligiöse - und mittlerweile in einflussreichen Kreisen der Computerindustrie populäre (vgl. STOA 2009) - Vision einer bevorstehenden radikalen Veränderung der „,conditio humana" durch Wissenschaft und Technik. Diese Vorstellung ist eine der derzeit erfolgreichsten Zukunftsvisionen des Transhumanismus, jener technokratisch-eschatologischen Weltanschauung, die den Diskursen über Nanotechnologie und dem Spezialdiskurs zu CT ihren Stempel aufgedrückt hat und überdies in der Debatte über „human enhancement" eine zentrale Rolle spielt (vgl. STOA 2009).

\section{Konturen des Konvergenzdiskurses}

Die „Expedition Zukunft“ kann, zusammengenommen mit der Sonderausgabe von „Spektrum der Wissenschaft", sinnbildlich für den CT-Diskurs stehen, wie ihn seine Initiatoren in den USA und ihr transhumanistisches Umfeld ursprünglich angelegt hatten. Die konvergenzwissenschaftliche und -technische Revolution ist demnach die Basis für einen tiefgreifenden Wandel in globalem Maßstab, im Zuge dessen letztlich die "conditio humana“ radikal transformiert und womöglich eine trans- oder posthumanistische Zeit eingeläutet wird. Eher im 
Vorübergehen als vorrangig sollen dabei die dringlichsten Probleme der Menschheit gelöst werden.

Realitätsnahen und wenig kontroversen Technikvisionen traut man anscheinend auch in der deutschen Ausstellung nicht zu, allein ausreichend Begeisterung für Wissenschaft und Technik erzeugen zu können, und wohl daher bedient man sich aus dem Ideen- und Bilderarsenal der transhumanistischen Technikvisionäre. Der extreme Technofuturismus wird durch die gleichzeitigen Verheißungen, globale Probleme bald lösen zu können, sowie unter häufigem Verweis auf (vermeintliche oder tatsächliche) Bedürfnisse von Kranken und Behinderten aufgewertet. Bedenkliche ethische, soziale oder politische Implikationen technologischer Konvergenz sowie Zweifel am Realitätssinn der Visionäre werden nur am Rande - und immer eng verbunden mit weit reichenden Verheißungen - thematisiert.

Zugleich haben sich aber auch neuere Entwicklungen niedergeschlagen: Das Label „Konvergenz" dient hier - ähnlich wie in politischen Programmen, bei Teilen der TA und auf einigen akademischen Konferenzen - als Chiffre für „Cutting-Edge“-Forschung und Technologieentwicklung mit noch unabsehbaren, aber vermutlich radikal einschneidenden Folgen. Dabei spiegelt die Ausstellung zudem wider, dass die Konvergenzbegrifflichkeit im Diskurs zur Synthetischen Biologie an Bedeutung gewonnen hat.

\section{Konvergenzdiskurs und Begleitforschung}

Vor diesem Hintergrund erscheint es an der Zeit, den CT-Spezialdiskurs in seinen neu entstandenen Kontexten zu betrachten. In diesen wird „Konvergenz" oft ebenfalls - ähnlich wie von der NBIC-Initiative und den Transhumanisten als eine Art Zauberwort genutzt, mit dem eine beeindruckende, zum Teil auch unheimliche, auf jeden Fall aber radikal neue Zukunft beschworen werden soll. Anstatt sich in der Begleitforschung immer wieder von Neuem auf die Suche nach dem „wahren“ (wissenschaftlich-technischen) „Kern“ der diversen visionären Programme $\mathrm{zu}$ machen, ist es womöglich zweckmäßiger, zunächst die Faszination durch Konvergenzvisionen übergreifend zu analysieren. $\mathrm{Zu}$ diesem Zweck bietet es sich zuvörderst an, die
Akteurskonstellationen (durchaus im Detail) sowie wiederkehrende Muster in den Themenkarrieren der verschiedenen Phänomene (wie „die Nanotechnologie“, „CT“ oder „Synthetische Biologie") zu betrachten. Von besonderem Interesse sind hier Überschneidungen zwischen den Einzeldiskursen.

Zudem wären hier auch ideengeschichtliche Aspekte zu berücksichtigen. Dabei verdient ein Ideensystem besondere Beachtung, das sich als „Ideologie extremen Fortschritts“ charakterisieren lässt und jüngst vor allem durch den Aufstieg des organisierten Transhumanismus wieder verstärkt Beachtung gefunden hat (vgl. dazu STOA 2009). Diese ursprünglich vor allem von visionären Naturwissenschaftlern entwickelte Ideologie ist im westlichen Haushalt der Ideen zu Wissenschaft, Technik und Gesellschaft fest verankert: Sie hat die für den heutigen Diskurs immer noch hoch relevanten Antiutopien des 20. Jahrhunderts und Sciencefiction stark geprägt. Die anthropologischen Grundannahmen dieser Ideologie laufen auf die Vision hinaus, zur extraterrestrischen Expansion befähigte Posthumane zu schaffen, wodurch u. a. Berührungspunkte zu Visionen aus der Militär- und Weltraumforschung bestehen. Als technokratische Eschatologie stellt sie eine Herausforderung für die christliche Theologie und für religiöse Menschen- und Weltbilder dar (dazu Coenen 2008). Schließlich erscheint das umfassende Kontrollparadigma, das insbesondere in den Publikationen der NBIC-Initiative deutlich wird, als eine radikalisierende Fortsetzung westlicher Ideen-, Wissenschafts- und Technikgeschichte.

Aufgrund ihrer sich abzeichnenden negativen Auswirkungen und ihrer Herkunft aus dem Wissenschaftssystem lässt sich mit Blick auf diese Ideologie von der Gefahr einer Zersetzung der Wissenschaft „von innen“ heraus sprechen. Dies betrifft ein zentrales Problem der Diskurse über CT, „human enhancement" und andere emergierende Phänomene, nämlich deren politisch-ideologisches Ungleichgewicht: An einem Ende des Spektrums, dem technik- und fortschrittskritischen, ist eine Barriere gegen unwissenschaftliche Glaubensüberzeugungen und fundamentalistische Strömungen aufgerichtet. Am anderen Ende des Spektrums, dem technikoptimistischen und Fortschritt bejahenden, sind hingegen die Grenzen zu Erlösungsideologien 
und mythischem Denken durchlässig. Dies dient objektiv zunächst dazu, Fundamentalopposition gegen technische Neuerungen zu schwächen offen aber ist, ob es auch dem Ziel der Förderung von Innovationen dient, da diese ja in erheblichem Maß auf gesellschaftliche Akzeptanz angewiesen sind. Wissenschaft dient unter dem auf innovative Anwendungen ausgerichteten technowissenschaftlichen Regime anscheinend in höherem Maß als früher letztlich dem Verkauf, und sie muss sich, gerade wo sie als zweckfreie oder Grundlagenforschung erscheint, selbst bewerben und verkaufen. Das Streben nach Erkenntnis und Wahrheit um ihrer selbst willen ist hier nicht einmal mehr regulatives Ideal, sondern höchstens noch schmückendes Beiwerk - wie auch der zweite Wagen im Ausstellungszug „Expedition Zukunft“ zeigt. Die zentralen Akteursgruppen im technowissenschaftlichen Regime, einschließlich der Naturwissenschaftler, Ingenieure und Begleitforscher, bedienen sich zwar auch dieses Beiwerks. Verfangen in einem Teufelskreis der Inflation von Versprechungen, wird für sie aber anscheinend der taktische Einsatz von Spekulationen (dazu Nordmann 2007), das technikvisionäre Marketing, immer attraktiver. Dieses kann eher altbacken daherkommen und bloß unfreiwillig provokant sein, wie bei einigen Schlüsselfiguren der NBIC-Initiative (denen man durchaus glauben mag, dass sie über die negativen Reaktionen auf ihre technokratischen Weltbeglückungsideen überrascht waren). Man stellt aber daneben avancierte Marketingstrategien fest, wie z. B. eine Art Schockwerbung („Shockvertising“), bei denen auch beängstigende Visionen einer extremen Zukunft dazu eingesetzt werden, um einerseits Aufmerksamkeit für die realen oder denkbaren destruktiven Potenziale bestimmter Technologiefelder zu schaffen und um andererseits Neuentwicklungen in diesen Technologiefeldern als förderungswürdige Mittel zur Weltrettung zu propagieren. ${ }^{6}$

Technikvisionäres Marketing trägt sowohl durch Heilsversprechen als auch durch Horrorvisionen zur Zersetzung wissenschaftlicher Rationalität bei. Insofern droht eine Zerstörung der Wissenschaft von innen heraus oder, genauer gesagt, eine Zerstörung der zunehmend marginalen, nach Erkenntnis strebenden Wissenschaft unter einem zu irrationaler Kommunikation neigenden technowissenschaftlichen Regime.
Der auch in den einschlägigen Diskursen zur Nanokonvergenz oft zitierte Satz Arthur C. Clarkes, dass jede ausreichend avancierte Technologie ununterscheidbar von Magie werde, liest sich vor diesem Hintergrund wie eine Drohung, dass der Fortschritt der „TechnoWissenschaft" zu einem Rückfall in ein magisches Zeitalter führen kann. Für die Begleitforschung besteht in diesem Zusammenhang die Gefahr, dass sie sich zur Magd der CT-Promotoren und ihrer Ideologen des extremen Fortschritts macht. Begleitforschung im Konvergenzdiskurs müsste indes vor allem der Versuch sein, suizidalen Tendenzen innerhalb der TechnoWissenschaft entgegenzuwirken.

\section{Anmerkungen}

1) $\mathrm{Zu}$ den ernüchternden Ergebnissen vgl. z. B. Andler et al. 2008 und Büscher in diesem Heft; zur spekulativen Ethik siehe Nordmann 2007 und $\mathrm{zu}$ den Human-Enhancement-Visionen vgl. STOA 2009.

2) Vgl. $\mathrm{zu}$ dieser Initiative vor allem deren ersten Bericht (Roco, Bainbridge 2002). „NBIC“" steht für „nano, bio, info, cogno“. Vgl. auch die Analysen zu den Aktivitäten der NBIC-Initiative und den europäischen Reaktionen auf diese in Andler et al. 2008 und TAB 2008 sowie den Beitrag von Büscher in diesem Heft.

3) Die folgende Analyse basiert im Wesentlichen auf einer Auswertung der Ausstellungsbegleittexte sowie einer Sonderausgabe einer populärwissenschaftlichen Zeitschrift (SdW 2009), die anlässlich der Ausstellung erschienen ist und auf dieser anstelle eines Ausstellungskatalogs verkauft wird. Die Sonderausgabe wurde maßgeblich von den Organisatoren der Ausstellung mitverantwortet. Die Analyse steht im Kontext einer langfristig angelegten systematischen Analyse und Bewertung von Visionen (,vision assessment") zu CT (vgl. z. B. TAB 2008; zum Instrument des ,vision assessment“ siehe z. B. Grin, Grunwald 2000; Grunwald 2004 und die kritische Analyse von Karafyllis 2009). Durch die Herkunft der CT-Debatte aus dem Diskurs über Nanotechnologie sowie wegen der Einbeziehung von Gestaltungselementen der Ausstellung in die Analyse stehen die folgenden Ausführungen auch im Kontext der Forschung zur Nutzung von Visionen und Bildern in der Kommunikation über Nanotechnologie (vgl. z. B. Lösch 2006).

4) Siehe zur Synthetischen Biologie auch Coenen/ Hennen/Link in diesem Heft.

5) Siehe zu dieser Thematik auch Coenen/Riehm 2008. 
6) Ein Beispiel dafür ist die katastrophenzählerische Befassung der Transhumanisten und anderer Technofuturisten mit sog. ,existenziellen Risiken“ (vgl. dazu Bostrom 2002).

7) Vgl. zu den verschiedenen Aspekten der „TechnoWissenschaft“ die Analyse von Nordmann 2005.

\section{Literatur}

Andler, D.; Barthelmé, S.; Beckert, B. et al., 2008: Converging Technologies and their Impact on the Social Sciences and Humanities. Final report of the CONTECS project, May 2008; http://www.contecs. fraunhofer.de/images/files/contecs_report_complete. pdf (download 4.9.09)

Bostrom, N., 2002: Existential Risks: Analyzing Human Extinction Scenarios and Related Hazards. In: Journal of Evolution and Technology 9/1 (2002) (ejournal; damals noch unter dem Titel „Journal of Transhumanism“); http://www.jetpress.org/volume 9/risks.html (download 4.9.09), o. S.

Coenen, C., 2008: Verbesserung des Menschen durch konvergierende Technologien - Christliche und posthumanistische Stimmen in einer aktuellen Technikdebatte. In: Böhm, H.; Ott, K. (Hg.): Bioethik - menschliche Identität in Grenzbereichen (Schriften der Evangelischen Forschungsakademie, Nr. 40). Leipzig, S. 41-123

Coenen, C.; Riehm, U., 2008: Entwicklung durch Vernetzung. Informations- und Kommunikationstechnologien in Afrika (Studien des Büros für Technikfolgen-Abschätzung beim Deutschen Bundestag, Bd. 26). Berlin

Grin, J.; Grunwald, A. (Hg.), 2000: Vision Assessment: Shaping Technology in $21^{\text {st }}$ Century Society. Berlin

Grunwald, A., 2004: Vision Assessment as a New Element of the FTA Toolbox. In: Scapolo, F.; Cahill, E. (Hg.): New Horizons and Challenges for Future-oriented Technology Analysis. Proceedings of the EU-US Scientific Seminar: New Technology Foresight, Forecasting \& Assessment Methods. European Commission DG JRC-IPTS Sevilla, 13.14.5.2004. Sevilla: European Communities 2005, S. 53-67 (Technical Report EUR 21473 EN - auch auf CD-ROM)

Grunwald, A., 2007: Orientierungsbedarf, Zukunftswissen und Naturalismus. Das Beispiel der ,technischen Verbesserung“" des Menschen. In: Deutsche Zeitschrift für Philosophie 55/6 (2007), S. 949-965

Karafyllis, N., 2009: Facts or Fiction? Methodological and Ethical Problems of Vision Assessment. In: Düwell, M.; Sollie, P. (Hg.): Evaluating New Technologies. Methodological Problems for the Ethical Assessment of Technologic Development (Library of Ethics and Applied Philosophy 3). Berlin, New York, S. 93-116

Kogge, W., 2008: Technologie des 21. Jahrhunderts. Perspektiven der Technikphilosophie (Literaturessay). In: Deutsche Zeitschrift für Philosophie 56/6 (2008), S. 935-956

Kurzweil, R., 2009: Der Mensch, Version 2.0. In: SdW, S. 121-126

Lösch, A., 2006: Anticipating the Futures of Nanotechnology: Visionary Images as Means of Communication. In: Technology Analysis \& Strategic Management Bd. 18, Heft 3/4 (Special Issue on the Sociology of Expectations in Science and Technology), S. 393-409

Nordmann, A., 2005: Was ist TechnoWissenschaft? - Zum Wandel der Wissenschaftskultur am Beispiel von Nanoforschung und Bionik. In: Rossmann, T.; Tropea, C. (Hg.): Bionik. Berlin, S. 209-218

Nordmann, A., 2007. If and Then: A Critique of Speculative NanoEthics. In: NanoEthics 1/1 (2007), S. 31-46

Roco, M.; Bainbridge, W. (Hg.), 2002: Converging Technologies for Improving Human Performance. Arlington; http://www.wtec.org/ConvergingTechno logies/1/NBIC_report.pdf (download 4.9.09)

SdW - Spektrum der Wissenschaft, 2009: Expedition Zukunft. Wie Forschung und Technik unser Leben verändern werden (Sonderausgabe in $\mathrm{Zu}$ sammenarbeit mit dem MPG-Projektteam „Expedition Zukunft"; April 2009). Heidelberg

STOA - Science and Technology Options Assessment, 2009: Human Enhancement (IP/A/STOA/ FWC/2005-28/SC35, $41 \&$ 45), IPOL/A/STOA/ 2007-13 (Mai 2009; Autoren: Coenen, C.; Schuijff, M.; Smits, M.; Klaasen, P.; Hennen, L.; Rader, M.; Wolbring, G.). Brüssel, Europäisches Parlament; http://www.europarl.europa.eu/stoa/publications/stud ies/stoa2007-13_en.pdf (download 4.9.09)

TAB - Büro für Technikfolgen-Abschätzung beim Deutschen Bundestag, 2008: Konvergierende Technologien und Wissenschaften (Autor: Coenen, Chr.), TAB-Hintergrundpapier Nr. 16. Berlin; http:/www.tab.fzk.de/de/projekt/zusammenfassung/ hp16.pdf (download 4.9.09)

\section{Kontakt}

Dipl.-Pol. Christopher Coenen Forschungszentrum Karlsruhe ITAS Postfach 36 40, 76021 Karlsruhe Tel.: +49 (0) 7247 / 82 - 4559 E-Mail: Christopher.Coenen@kit.edu 